\title{
Comparison of treatment outcome between living donor liver transplantation and sorafenib for patients with hepatocellular carcinoma beyond the Milan criteria
}

\author{
Yuri Cho ${ }^{1,3}$, Jeong-Hoon Lee ${ }^{1}$, Dong Hyeon Lee ${ }^{1,4}$, Eun Ju Cho ${ }^{1}$, Su Jong Yu ${ }^{1}$, Nam- \\ Joon $\mathrm{Yi}^{2}$, Kwang-Woong Lee ${ }^{2}$, Yoon Jun Kim ${ }^{1}$, Jung-Hwan Yoon ${ }^{1}$ and Kyung-Suk Suh ${ }^{2}$ \\ ${ }^{1}$ Department of Internal Medicine and Liver Research Institute, Seoul National University College of Medicine, Seoul, Republic \\ of Korea \\ ${ }^{2}$ Department of Surgery, Seoul National University College of Medicine, Seoul, Republic of Korea \\ ${ }^{3}$ Department of Internal Medicine, CHA Gangnam Medical Center, CHA University, Seoul, Republic of Korea \\ ${ }^{4}$ Department of Internal Medicine, Seoul Metropolitan Government Seoul National University Boramae Medical Center, Seoul, \\ Republic of Korea
}

Correspondence to: Jeong-Hoon Lee, email: pindra@empal.com

Keywords: hepatocellular carcinoma, living donor liver transplantation, sorafenib, MoRAL score, survival

Received: March 29, $2017 \quad$ Accepted: April 26, $2017 \quad$ Published: May 10, 2017

Copyright: Cho et al. This is an open-access article distributed under the terms of the Creative Commons Attribution License 3.0 (CC BY 3.0), which permits unrestricted use, distribution, and reproduction in any medium, provided the original author and source are credited.

\section{ABSTRACT}

For patients with advanced hepatocellular carcinoma (HCC), sorafenib is the only systemic treatment recommended by international guidelines. We recently reported that HCC patients with a low MoRAL (model to predict tumor recurrence after LDLT) score $(\leq \mathbf{3 1 4 . 8})$ have excellent treatment outcomes after living-donor liver transplantation (LDLT), even though they are beyond the Milan criteria. In the present study, we investigated whether LDLT offers a better treatment outcome than sorafenib for patients with HCC beyond the Milan criteria according to the MoRAL score. A retrospective cohort study of 325 consecutive patients who were treated with either LDLT $(n=122)$ or sorafenib $(n=203)$ for HCC beyond the Milan criteria from 2005 to 2014 at a tertiary hospital was performed. The primary and secondary endpoints were overall survival (OS) and time-to-progression. When baseline characteristics were balanced using inverse probability weighting, OS was significantly longer in the LDLT group than in the sorafenib group (5-year OS rate, $71.9 \%$ vs. $4.9 \% ; H R=0.1 ; P<0.001)$. The LDLT group exhibited a significantly lower risk of tumor progression (5-year recurrence rate, $34.7 \%$ vs. $96 \%$; HR=0.14; $P<0.001)$ than the sorafenib group. The increase in OS with LDLT was predominantly among patients with a low MoRAL score (5-year OS rate, $81.1 \%$ vs. $5.8 \%$; HR=0.06; $P<0.001)$ compared with those with a high MoRAL score (5-year OS rate, $28.3 \%$ vs. $4.3 \%$; HR $=0.42 ; P=0.047$ ). Patients with a low MoRAL score and without extrahepatic metastasis or hepatic vein invasion might be good candidates for LDLT instead of sorafenib treatment if there is a willing living related donor.

\section{INTRODUCTION}

Liver transplantation has been widely accepted as the treatment of choice for both the early stages of hepatocellular carcinoma (HCC) [1] and end-stage liver disease [2]. Recently, there has been a gradual expansion of the liver transplantation recipient criteria due to excellent overall survival (OS). Expanding the selection criteria for patients beyond the Milan criteria has been suggested by some pioneers, resulting in more patients who are beyond the Milan criteria being cured by liver transplantation at the expense of a higher rate of recurrence. Patients with HCC exceeding the Milan criteria have shown a high recurrence rate after liver transplantation - up to $50 \%$ at 3 years [3]. However, some of these patients with favorable tumor biology achieve 
long-term recurrence-free-survival and are probably cured; these patients might be good candidates for living-donor liver transplantation (LDLT) [4-6].

In Asia, LDLT is more frequently performed than deceased-donor liver transplantation (DDLT) due to a shortage of deceased donors and possibly also for cultural and religious reasons [7]. Therefore, guidelines detailing how to select recipient candidates for LDLT in advanced HCC are essential. We previously established and externally validated a model to predict tumor recurrence after LDLT for HCC beyond the Milan criteria: the model to predict tumor recurrence after LDLT (MoRAL) score. The MoRAL score uses serum alpha-fetoprotein (AFP) and protein induced by vitamin $\mathrm{K}$ absence-II (PIVKA-II) levels in the formula: $11 \times \sqrt{ }$ PIVKA +2 $\times \sqrt{ }$ AFP. The score can predict tumor recurrence after LDLT and identifies those patients with the potential for low recurrence and long-term OS. The 5-year OS and the 5-year cumulative tumor recurrence rates are approximately $80 \%$ and $30 \%$, respectively, in patients beyond the Milan criteria with a low MoRAL score $(\leq 314.8)$ who might be good candidates for LDLT [8].

For patients with advanced HCC, sorafenib is the only treatment proven to have a survival benefit over the best supportive care. However, the median survival and the time-to-radiologic progression are only 2.3 to 3 months longer for patients treated with sorafenib than for those given placebo. The median OS was 10.7 months in the sorafenib group in the Phase III Study of Sorafenib in Patients With Advanced Hepatocellular Carcinoma (SHARP trial) [9, 10]. No consistent survival benefit has been reported for other anticancer systemic therapeutic agents for HCC in approximately 100 randomized studies that investigated intraarterial systemic chemotherapy (doxorubicin and platinum), various hormonal therapies (tamoxifen and antiandrogens), and immunotherapy (interferon alfa) [11-14]. Although locoregional therapies (e.g., radiofrequency ablation, percutaneous ethanol injection, and transarterial chemoembolization) or radiotherapy (conformal or stereotactic) are recommended for patients with unresectable HCC $[15,16]$, only a few of these are proven to have a survival benefit greater than 1 year $[17,18]$. The patients' underlying poor liver function often limits the utility of locoregional therapies due to the risk of liver failure after treatment. Therefore, for patients with unresectable HCC, LDLT is considered an alternative treatment option.

To date, there has been no study analyzing the survival benefit of LDLT over sorafenib for patients beyond the Milan criteria. The present study analyzes whether LDLT offers a better treatment outcome than sorafenib for HCC patients beyond the Milan criteria, according to their MoRAL scores.

\section{RESULTS}

\section{Baseline characteristics}

A total of 325 consecutive patients (122 patients in the LDLT group, 203 patients in the sorafenib group) were analyzed. Table 1 shows the baseline characteristics of the two groups. There were significant differences in the baseline age, Child-Pugh score, and American Joint Committee on Cancer (AJCC), 7th edition, T classification between groups.

The median age was 59 years in the LDLT group and 63 years in the sorafenib group $(P=0.01)$. Hepatitis $\mathrm{B}$ virus was the most common etiology of $\mathrm{HCC}$ in both groups. Only $2.5 \%$ of patients in the sorafenib group were Child-Pugh class C, less than the $22.1 \%$ in the LDLT group $(P<0.001)$. Forty-seven patients $(38.5 \%)$ in the LDLT group and 80 patients $(39.4 \%)$ in the sorafenib group had portal vein invasion, respectively $(P=0.91)$. After inverse probability weighting (IPW), 60 patients $(49.2 \%)$ in the LDLT group and 80 patients $(39.4 \%)$ in the sorafenib group had portal vein invasion, respectively $(P=0.13)$.

The median serum AFP and PIVKA-II levels were higher in the sorafenib group than in the LDLT group. The median MoRAL score in the sorafenib group was higher than that of the LDLT group (266.7 vs. 113.2; $P<0.001)$. The median wait time prior to LDLT (from HCC diagnosis to LDLT) was 6.9 months (range, 0.3-131.1 months). There was no donor mortality.

\section{Comparison between LDLT and sorafenib groups for OS and time-to-progression (TTP)}

After IPW, the baseline characteristics, including age, AJCC T classification, and Child-Pugh class, became more balanced than before weighting and did not differ significantly between groups (Table 2).

When the baseline characteristics were balanced using IPW, the LDLT group showed a significantly lower risk of death than the sorafenib group (Figure 1A; hazard ratio $[\mathrm{HR}]=0.1 ; 95 \%$ confidence interval $[\mathrm{CI}], 0.05-0.2$; $P<0.001)$. The median OS of the sorafenib group was 8.3 months (interquartile range [IQR], 3.2-18.2 months), while that of the LDLT group was not reached (IQR, 34.1 months-not applicable). The 5-year OS rate was $71.9 \%$ in the LDLT group and $4.9 \%$ in the sorafenib group. The LDLT group also had a lower risk of tumor progression (Figure 1B; HR $=0.14 ; 95 \% \mathrm{CI}, 0.08-0.24 ; P<0.001$ ). The 5-year cumulative tumor recurrence rate was $34.7 \%$ in the LDLT group and $96.0 \%$ in the sorafenib group.

Multivariate Cox regression analyses for OS (Table 3) and TTP (Table 4) was performed before and after IPW. The independent predictors of OS and TTP 
Table 1: Baseline characteristics

\begin{tabular}{|c|c|c|c|}
\hline Clinical characteristics & LDLT group $(n=122)$ & Sorafenib group $(n=203)$ & $P$-value \\
\hline Age (years) & $59(35-75)$ & $63(40-86)$ & 0.01 \\
\hline Male, No. (\%) & $104(85.2 \%)$ & $179(88.2 \%)$ & 0.65 \\
\hline $\begin{array}{l}\text { Etiology, No. (\%) } \\
\text { HBV/HCV/alcohol/others }\end{array}$ & $\begin{array}{c}100 / 13 / 5 / 4 \\
(82.0 / 10.7 / 4.1 / 3.3 \%)\end{array}$ & $\begin{array}{c}145 / 32 / 12 / 14 \\
(71.4 / 15.8 / 5.9 / 6.9 \%)\end{array}$ & 0.26 \\
\hline $\begin{array}{l}\text { Child-Pugh class, No. (\%) } \\
\text { A/B/C }\end{array}$ & $\begin{array}{c}50 / 45 / 27 \\
(41.0 / 36.9 / 22.1 \%)\end{array}$ & $\begin{array}{c}64 / 134 / 5 \\
(31.5 / 66.0 / 2.5 \%)\end{array}$ & $<0.001$ \\
\hline $\begin{array}{l}\text { BCLC stage, No. (\%) } \\
\text { B/C/D }\end{array}$ & $\begin{array}{c}60 / 35 / 27 \\
(49.2 / 28.7 / 22.1 \%)\end{array}$ & $\begin{array}{c}108 / 90 / 5 \\
(53.2 / 44.3 / 2.5 \%)\end{array}$ & $<0.001$ \\
\hline $\begin{array}{l}\text { AJCC 7th T classification } \\
\text { T1 } \\
\text { T2 } \\
\text { T3a } \\
\text { T3b } \\
\text { T4 }\end{array}$ & $\begin{array}{c}0 \\
25(22.7 \%) \\
29(26.4 \%) \\
20(18.2 \%) \\
36(32.7 \%)\end{array}$ & $\begin{array}{c}2(1.0 \%) \\
64(31.5 \%) \\
24(13.3 \%) \\
86(47.8 \%) \\
4(2.2 \%)\end{array}$ & $<0.001$ \\
\hline MELD score & $8.9(2.5-22.6)$ & $6.9(1.2-14.5)$ & $<0.001$ \\
\hline AFP (ng/mL) & $21.3(1.3-1708000)$ & $212.3(1.3-427000)$ & $<0.001$ \\
\hline PIVKA-II (mAU/mL) & $57(6-75000)$ & $308(2.9-75000)$ & $<0.001$ \\
\hline Maximal tumor size $(\mathrm{cm})$ & $2.9(1.2-13.8)$ & $5(1-10)$ & $<0.001$ \\
\hline Number of nodules & $4(1-30)$ & $5(1-28)$ & 0.42 \\
\hline $\begin{array}{l}\text { Type of HCC, No. (\%) } \\
\text { Nodular/Diffuse or infiltrative }\end{array}$ & $99 / 23(81.1 / 18.9 \%)$ & $153 / 50(75.4 / 24.6 \%)$ & 0.27 \\
\hline Portal vein invasion, No. (\%) & $47(38.5 \%)$ & $80(39.4 \%)$ & 0.91 \\
\hline $\begin{array}{l}\text { Location of portal vein invasion, No. (\%) } \\
\text { Intrahepatic } \\
\text { Extrahepatic }\end{array}$ & $\begin{array}{l}28(59.6 \%) \\
19(40.4 \%)\end{array}$ & $\begin{array}{l}37(46.3 \%) \\
43(53.7 \%)\end{array}$ & 0.15 \\
\hline MoRAL score & $113.2(33.7-3928.3)$ & $266.7(14.1-3969.3)$ & $<0.001$ \\
\hline
\end{tabular}

Data are expressed as $\mathrm{n}(\%)$ or median with range.

Abbreviation: LDLT, living donor liver transplantation; HBV, hepatitis B virus; $\mathrm{HCV}$, hepatitis $\mathrm{C}$ virus; $\mathrm{BCLC}$, Barcelona clinic liver cancer; AJCC, American Joint Committee on Cancer; MELD, Model For End-Stage Liver Disease; AFP, alphafetoprotein; PIVKA-II protein induced by vitamin K absence-II; HCC, hepatocellular carcinoma; MoRAL, model to predict tumor recurrence after LDLT.

included the AJCC T classification and the treatment group (LDLT vs. sorafenib). The treatment group was the most significant predictor of both OS and TTP.

When we performed subgroup analysis for the patients with portal vein invasion, the LDLT group still showed a significantly lower risk of death (Figure 2A; HR $=0.11 ; 95 \% \mathrm{CI}, 0.05-0.27 ; P<0.001)$ and a significantly lower risk of tumor recurrence (Figure 2B; HR $=0.2 ; 95 \%$ CI, $0.1-0.39 ; P<0.001)$ than the sorafenib group.

\section{Subgroup analysis according to the MoRAL score for OS and TTP}

We performed subgroup analysis by dividing patients into two groups according to the MoRAL score, using a cutoff of 314.8 . We then compared the LDLT and sorafenib group for OS and TTP.
The increase in OS for LDLT over sorafenib was more predominant in those patients with a low MoRAL score $(\leq 314.8)$ (Figure 3A; HR $=0.06$; 95\% CI, $0.02-0.17 ; P<0.001)$ than in those with a high MoRAL score (> 314.8) (Figure 3B; HR $=0.41 ; 95 \%$ CI, $0.18-0.99 ; P=0.002)$. Among the patients with a low MoRAL score, the median OS was not reached in the LDLT group and 13.7 months (IQR, 6.1-28.7 months) in the sorafenib group. The 5-year OS rate was $81.1 \%$ in the LDLT group with a low MoRAL score and 5.8\% in the sorafenib group with a low MoRAL score. In patients with a high MoRAL score, the median OS was 16.0 months (IQR, 6.6-71.0 months) in the LDLT group and 4.3 months (IQR, 2.8-9.8 months) in the sorafenib group. The 5-year OS rate was $28.3 \%$ in the LDLT group with a high MoRAL score and $4.3 \%$ in the sorafenib group with a high MoRAL score. 


\begin{tabular}{|c|c|c|c|c|c|c|c|c|}
\hline & \multicolumn{2}{|c|}{ LDLT group } & \multicolumn{2}{|c|}{$\begin{array}{l}\text { Sorafenib } \\
\text { group }\end{array}$} & \multirow{2}{*}{$\begin{array}{c}\text { Standardized } \\
\text { Effect Size } \\
\text { (unweighted) }\end{array}$} & \multirow{2}{*}{$\begin{array}{c}P \text {-Value } \\
\text { (unweighted) }\end{array}$} & \multirow{2}{*}{$\begin{array}{c}\text { Standardized } \\
\text { Effect Size } \\
\text { (weighted) }\end{array}$} & \multirow{2}{*}{$\begin{array}{c}P \text {-Value } \\
\text { (weighted) }\end{array}$} \\
\hline & Mean & SD & Mean & SD & & & & \\
\hline Age (years) & 58.6 & 7.98 & 62.68 & 10.73 & -0.432 & 0.04 & -0.202 & 0.46 \\
\hline Sex & & & & & & 0.21 & & 0.30 \\
\hline Male & 0.85 & 0.4 & 0.89 & 0.31 & -0.262 & & 0.174 & \\
\hline Female & 0.15 & 0.4 & 0.11 & 0.31 & 0.262 & & -0.174 & \\
\hline AJCC 7th T classification & & & & & & $<0.001$ & & 0.97 \\
\hline $\mathrm{T} 2$ & 0.08 & 0.27 & 0.21 & 0.41 & -0.382 & & -0.095 & \\
\hline $\mathrm{T} 3 \mathrm{a}$ & 0.12 & 0.32 & 0.13 & 0.33 & -0.023 & & -0.099 & \\
\hline $\mathrm{T} 3 \mathrm{~b}$ & 0.24 & 0.43 & 0.63 & 0.48 & -0.850 & & 0.117 & \\
\hline $\mathrm{T} 4$ & 0.56 & 0.5 & 0.02 & 0.14 & 1.474 & & 0.053 & \\
\hline Child Pugh class & & & & & & $<0.001$ & & 0.93 \\
\hline A & 0.16 & 0.37 & 0.37 & 0.48 & -0.495 & & -0.002 & \\
\hline $\mathrm{B}$ & 0.52 & 0.5 & 0.59 & 0.49 & -0.131 & & -0.058 & \\
\hline $\mathrm{C}$ & 0.32 & 0.47 & 0.04 & 0.2 & 0.772 & & 0.111 & \\
\hline
\end{tabular}

Abbreviation: LDLT, living donor liver transplantation; SD, standard deviation; AJCC, American Joint Committee on Cancer.

The increase in TTP for LDLT over sorafenib was also more predominant in those patients with a low MoRAL score (Figure 4A; HR $=0.08 ; 95 \% \mathrm{CI}$, $0.04-0.16 ; P<0.001)$ than in those with a high MoRAL score (Figure 4B; HR $=0.53 ; 95 \%$ CI, 0.28-0.91; $P=0.03)$. Among the patients with a low MoRAL score, the median TTP was not reached in the LDLT group and
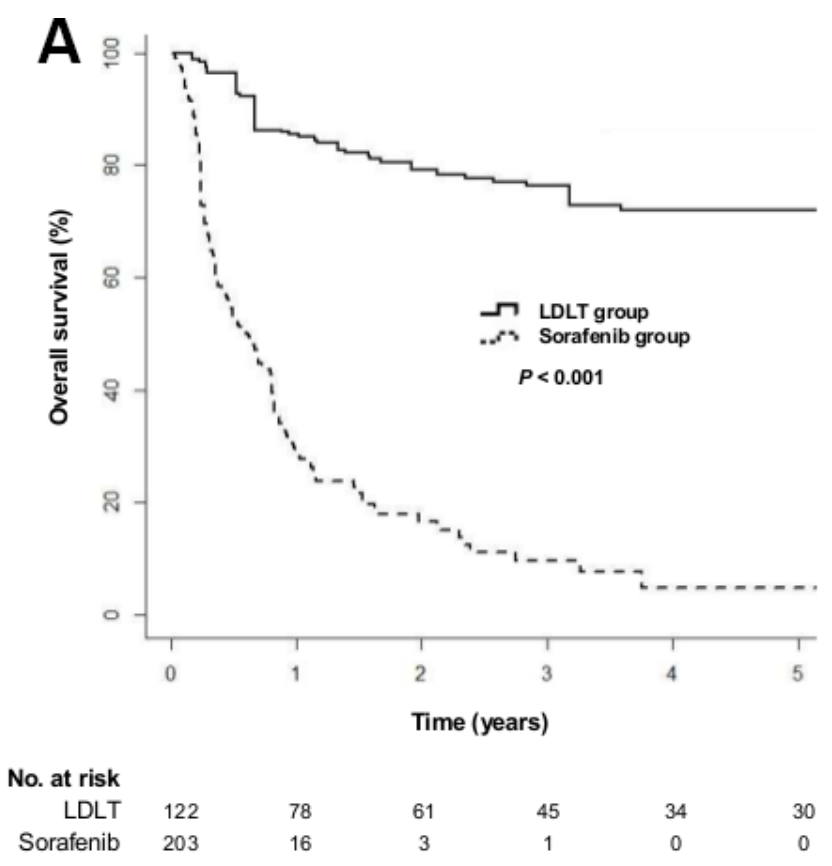

4.1 months (IQR, 1.6-7.7 months) in the sorafenib group. The 5-year tumor recurrence rate in the LDLT group with a low MoRAL score was only $22.2 \%$, while that in the sorafenib group with a low MoRAL score was $96.8 \%$. In patients with a high MoRAL score, the median TTP was 7.4 months (IQR, 4.0-32.8 months) in the LDLT group and 3.7 months (IQR, 1.4-7.4 months) in the sorafenib
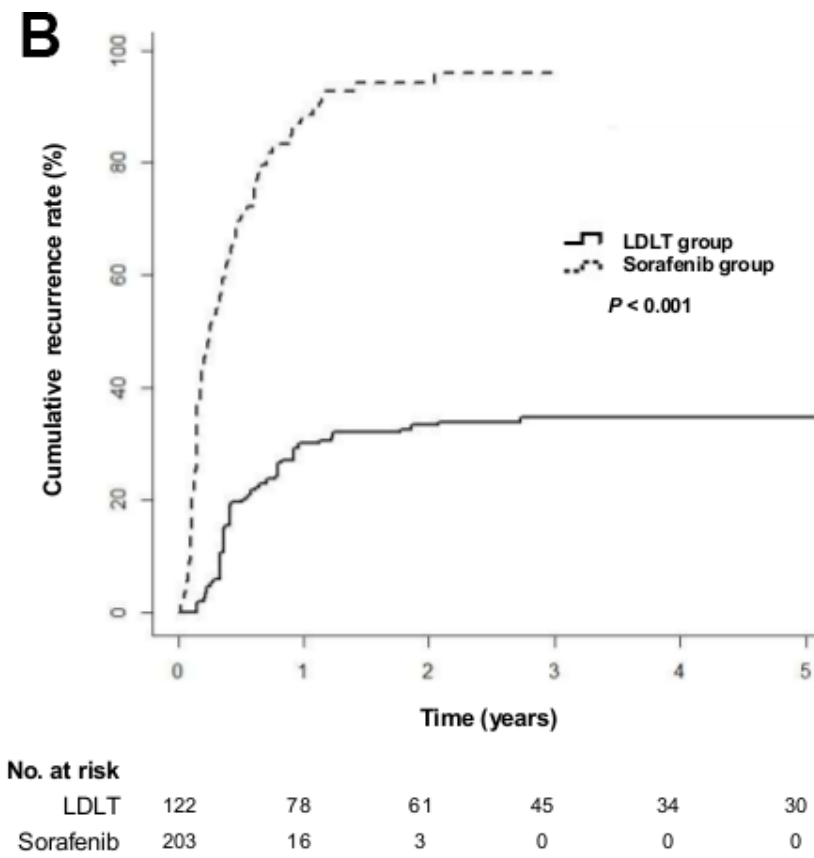

Figure 1: Cumulative overall survival and tumor recurrence rates in the living-donor liver transplantation (LDLT) and sorafenib groups after inverse probability weighting (IPW). (A) Cumulative overall survival rates $(P<0.001$ by log-rank test). (B) Cumulative tumor-recurrence rates $(P<0.001$ by log-rank test). 
Table 3: Univariate and multivariate Cox proportional hazard analyses for overall survival

\begin{tabular}{|c|c|c|c|c|}
\hline \multirow[b]{2}{*}{ Variable } & \multicolumn{2}{|c|}{ Univariate analysis } & \multicolumn{2}{|c|}{ Multivariate analysis } \\
\hline & HR (95\% CI) & $P$-value & HR (95\% CI) & $P$-value \\
\hline Age (years) & $1.01(1.0-1.03)$ & 0.13 & & \\
\hline Sex, female ( $v s$. male) & $0.84(0.51-1.39)$ & 0.51 & & \\
\hline $\begin{array}{l}\text { AJCC 7th T classification (vs. T1) } \\
\text { T2 } \\
\text { T3a } \\
\text { T3b } \\
\text { T4 }\end{array}$ & $\begin{array}{c}0.13(0.03-0.52) \\
0.13(0.03-0.54) \\
0.30(0.07-1.21) \\
0.09(0.02-0.41)\end{array}$ & $\begin{array}{c}0.004 \\
0.005 \\
0.09 \\
0.002\end{array}$ & $\begin{array}{c}0.14(0.03-0.60) \\
0.37(0.09-1.59) \\
0.37(0.09-1.51) \\
0.59(0.13-2.68)\end{array}$ & $\begin{array}{l}0.01 \\
0.18 \\
0.16 \\
0.50\end{array}$ \\
\hline $\begin{array}{l}\text { Child-Pugh class (vs. A) } \\
\text { B } \\
\text { C }\end{array}$ & $\begin{array}{l}1.27(0.90-1.80) \\
0.45(0.23-0.86)\end{array}$ & $\begin{array}{l}0.17 \\
0.02\end{array}$ & $\begin{array}{l}0.87(0.61-1.23) \\
0.99(0.47-2.11)\end{array}$ & $\begin{array}{l}0.43 \\
0.98\end{array}$ \\
\hline $\begin{array}{l}\text { Treatment group ( } v s . \text { sorafenib group) } \\
\text { LDLT group }\end{array}$ & $0.12(0.08-0.19)$ & $<0.001$ & $0.07(0.04-0.13)$ & $<0.001$ \\
\hline After IPW & & & & \\
\hline Age (years) & $0.99(0.95-1.02)$ & 0.50 & & \\
\hline Sex, female (vs. male) & $1.15(0.63-2.07)$ & 0.65 & & \\
\hline $\begin{array}{l}\text { AJCC 7th T classification (vs. T1) } \\
\text { T2 } \\
\text { T3a } \\
\text { T3b } \\
\text { T4 }\end{array}$ & $\begin{array}{l}0.11(0.03-0.43) \\
0.17(0.04-0.72) \\
0.20(0.05-0.79) \\
0.22(0.05-0.99)\end{array}$ & $\begin{array}{l}0.002 \\
0.02 \\
0.02 \\
0.04\end{array}$ & $\begin{array}{l}0.17(0.04-0.67) \\
0.42(0.10-1.76) \\
0.44(0.11-1.76) \\
0.51(0.11-2.31)\end{array}$ & $\begin{array}{l}0.01 \\
0.24 \\
0.25 \\
0.38\end{array}$ \\
\hline $\begin{array}{l}\text { Child-Pugh class (vs. A) } \\
\text { B } \\
\text { C }\end{array}$ & $\begin{array}{l}0.90(0.53-1.54) \\
0.74(0.34-1.61)\end{array}$ & $\begin{array}{l}0.70 \\
0.45\end{array}$ & & \\
\hline $\begin{array}{l}\text { Treatment group (vs. sorafenib group) } \\
\text { LDLT group }\end{array}$ & $0.12(0.06-0.23)$ & $<0.001$ & $0.10(0.05-0.20)$ & $<0.001$ \\
\hline
\end{tabular}

Abbreviation: HR, hazard ratio; CI, confidence interval; AJCC, American Joint Committee on Cancer; LDLT, living donor liver transplantation; IPW, inverse probability weighting

group. The 5-year cumulative tumor recurrence rate in the LDLT group with a high MoRAL score was $91.6 \%$, while that in the sorafenib group with a high MoRAL score was $95.4 \%$.

\section{DISCUSSION}

In this study, we found that LDLT confers significantly longer OS and TTP than sorafenib for patients with HCC beyond the Milan criteria. The increase in OS for LDLT over sorafenib is more predominant in those patients with a low MoRAL score $(\leq 314.8)$. Our results suggest that patients beyond the Milan criteria with a low MoRAL score and without extrahepatic metastasis might be good candidates for LDLT rather than sorafenib treatment, if there is a willing living related donor.

Sorafenib is a multitargeted tyrosine kinase inhibitor that inhibits vascular endothelial growth factor receptor, platelet-derived growth factor receptor, b-Raf, Fms-related tyrosine kinase, and c-kit [19]. Sorafenib has limited efficacy and a low objective tumor response rate (partial response rate $=2 \%$, stable disease rate $=40 \%)[9,20]$. However, sorafenib is still the standard treatment when patients have extrahepatic metastasis or unresectable HCC that is either not suitable for or refractory to transarterial chemoembolization. Approximately half of patients with $\mathrm{HCC}$ are candidates for systemic chemotherapy, including sorafenib [21,22]. The prognosis of those patients is poor, with less than 8 months of OS if untreated. Due to the limited efficacy of sorafenib, there is an urgent need to look beyond this medication and establish a more effective therapy for HCC. According to the Hong Kong Liver Cancer staging criteria, surgical- or locoregional treatment is recommend for the subgroup of patients with advanced HCC, such as multinodular or locally-advanced HCC, if they have preserved liver function [23].

The Milan criteria are considered a universal standard for selecting HCC patients for liver transplantation, limiting the risk of tumor recurrence to an acceptable level [1,24]. However, the feasibility of liver transplantation for a patient beyond the Milan criteria is still a topic of debate. Although the recurrence rate is high, liver transplantation remains 
the only possible curative treatment for these patients. Therefore, we analyzed the efficacy of LDLT versus sorafenib for these patients. Liver transplantation provides oncologic and cirrhotic liver clearance. In this regard, liver transplantation differs from other locoregional treatments or systemic chemotherapy. In this study, we found that LDLT might be a successful strategy to prolong OS and TTP for those patients. To our knowledge, this is the first report showing a significant survival benefit for LDLT over sorafenib for patients with HCC beyond the Milan criteria.
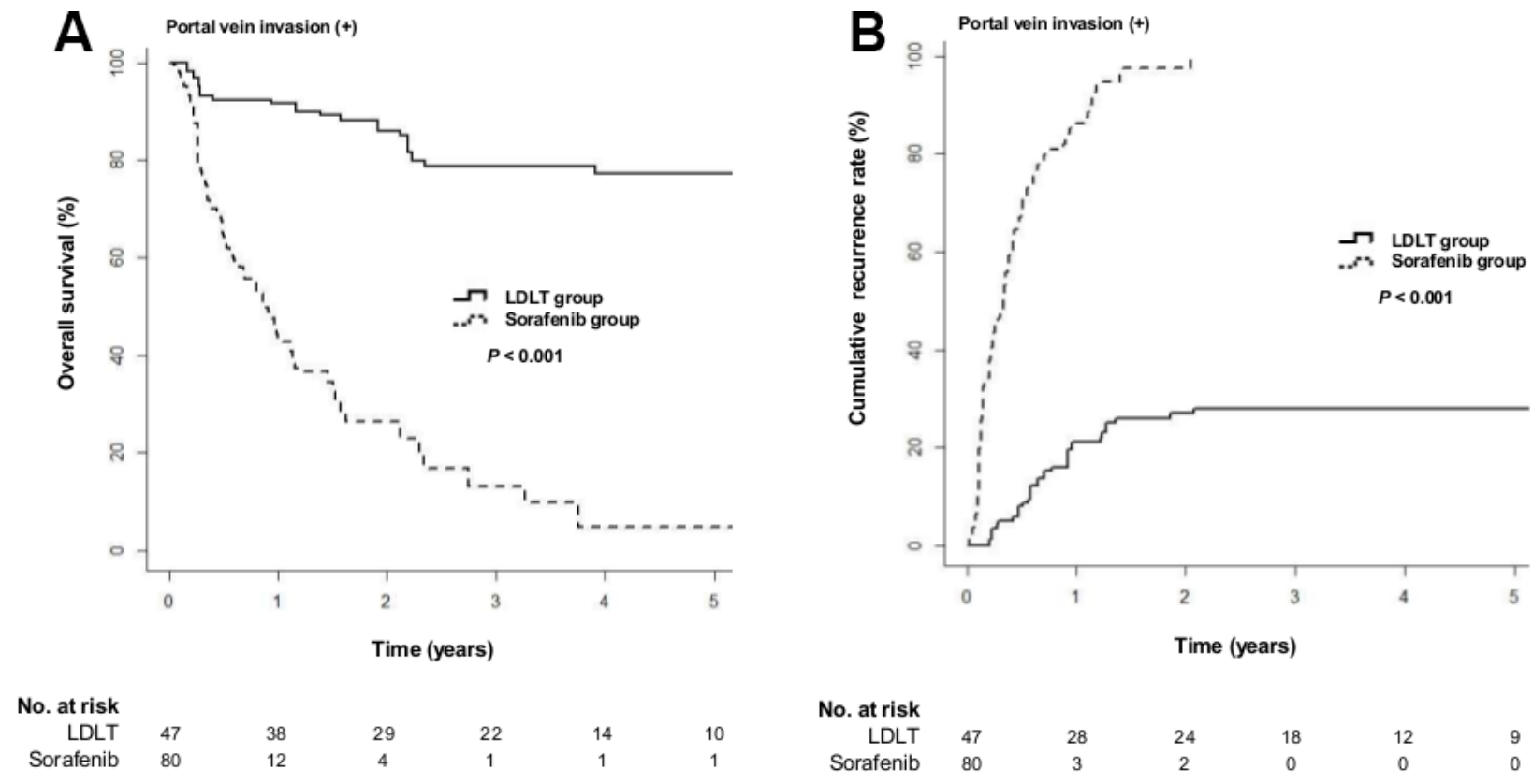

Figure 2: Cumulative overall survival and tumor recurrence rates in the LDLT and sorafenib groups after IPW for the patients with portal vein invasion. (A) Cumulative overall survival rates $(P<0.001$ by log-rank test). (B) Cumulative tumor recurrence rates $(P<0.001$ by log-rank test $)$.
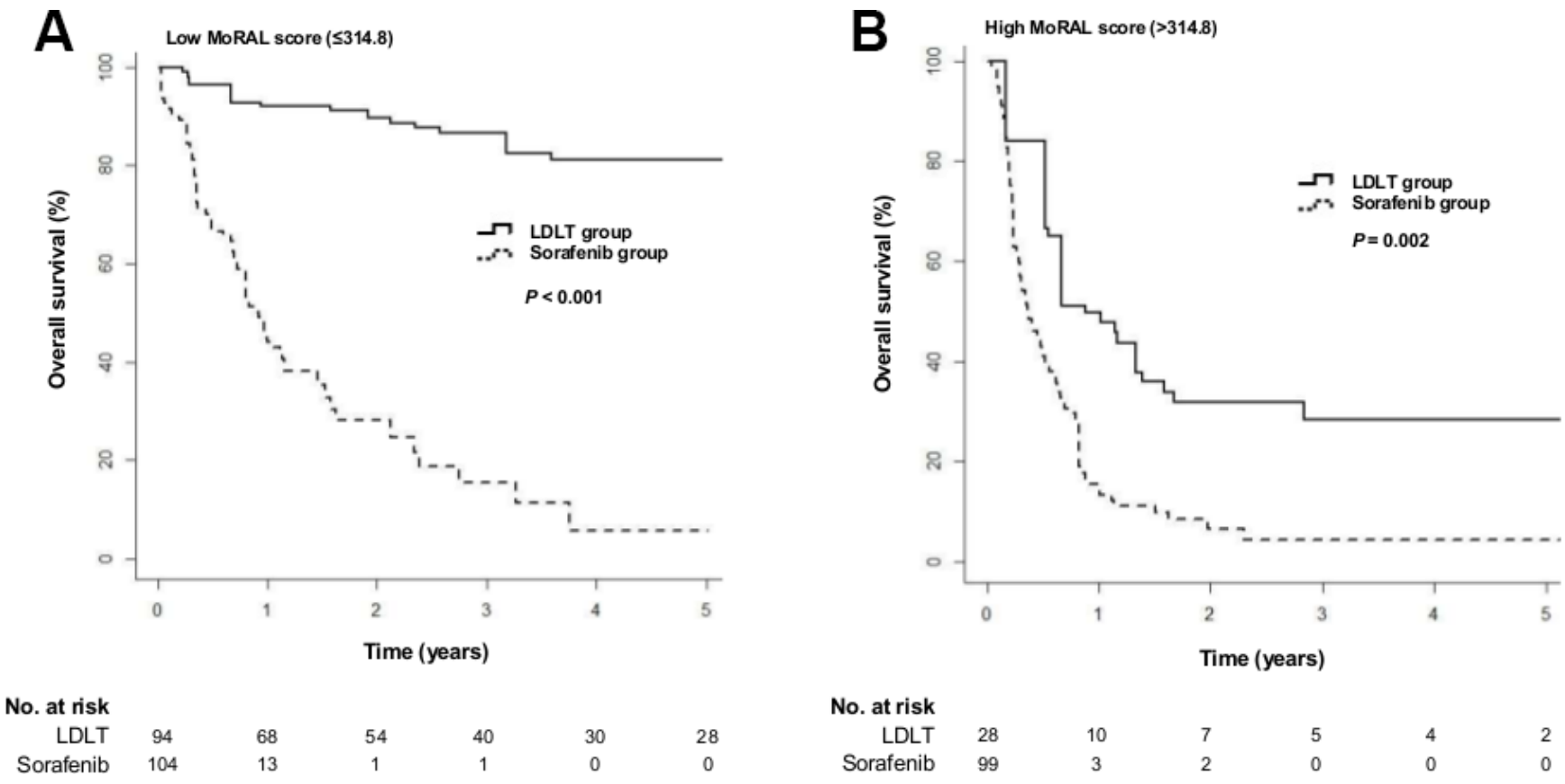

Figure 3: Cumulative overall survival in the LDLT and sorafenib groups according to the MoRAL score using a cutoff of 314.8. (A) Cumulative overall survival in patients with a low MoRAL score $(\leq 314.8 ; P<0.001$ by log-rank test). (B) Cumulative overall survival in patients with a high MoRAL score ( $>314.8 ; P=0.002$ by log-rank test) 
Table 4: Univariate and multivariate Cox proportional hazard analyses for time-to-progression

\begin{tabular}{|c|c|c|c|c|}
\hline \multirow[b]{2}{*}{ Variable } & \multicolumn{2}{|c|}{ Univariate analysis } & \multicolumn{2}{|c|}{ Multivariate analysis } \\
\hline & HR (95\% CI) & $P$-value & HR (95\% CI) & $P$-value \\
\hline Age (years) & $1.01(1.0-1.03)$ & 0.25 & & \\
\hline Sex, female ( $v s$. male) & $0.78(0.48-1.28)$ & 0.33 & & \\
\hline $\begin{array}{l}\text { AJCC 7th T classification (vs. T1) } \\
\text { T2 } \\
\text { T3a } \\
\text { T3b } \\
\text { T4 }\end{array}$ & $\begin{array}{c}0.59(0.08-4.24) \\
0.28(0.04-2.13) \\
0.72(0.10-5.2) \\
0.38(0.05-2.8)\end{array}$ & $\begin{array}{l}0.60 \\
0.22 \\
0.74 \\
0.34\end{array}$ & & \\
\hline $\begin{array}{l}\text { Child-Pugh class (vs. A) } \\
\text { B } \\
\text { C }\end{array}$ & $\begin{array}{c}1.5(1.07-2.1) \\
0.39(0.19-0.79)\end{array}$ & $\begin{array}{l}0.02 \\
0.01\end{array}$ & $\begin{array}{l}1.08(0.77-1.52) \\
0.82(0.39-1.76)\end{array}$ & $\begin{array}{l}0.66 \\
0.62\end{array}$ \\
\hline $\begin{array}{l}\text { Treatment group (vs. sorafenib } \\
\text { group) } \\
\text { LDLT group }\end{array}$ & $0.15(0.10-0.22)$ & $<0.001$ & $0.16(0.10-0.25)$ & $<0.001$ \\
\hline After IPW & & & & \\
\hline Age (years) & $0.99(0.95-1.02)$ & 0.39 & & \\
\hline Sex, female ( $v s$. male) & $0.94(0.50-1.76)$ & 0.83 & & \\
\hline $\begin{array}{l}\text { AJCC 7th T classification (vs. T1) } \\
\text { T2 } \\
\text { T3a } \\
\text { T3b } \\
\text { T4 }\end{array}$ & $\begin{array}{l}0.49(0.35-0.68) \\
0.36(0.21-0.61) \\
0.54(0.34-0.84) \\
0.86(0.48-1.53)\end{array}$ & $\begin{array}{c}<0.001 \\
<0.001 \\
0.01 \\
0.61\end{array}$ & $\begin{array}{l}0.94(0.70-1.28) \\
0.99(0.59-1.69) \\
1.29(0.86-1.94) \\
2.33(1.29-4.23)\end{array}$ & $\begin{array}{l}0.70 \\
1.00 \\
0.22 \\
0.01\end{array}$ \\
\hline $\begin{array}{l}\text { Child-Pugh class (vs. A) } \\
\text { B } \\
\text { C }\end{array}$ & $\begin{array}{l}1.19(0.70-2.05) \\
0.64(0.28-1.46)\end{array}$ & $\begin{array}{l}0.52 \\
0.29\end{array}$ & & \\
\hline $\begin{array}{l}\text { Treatment group (vs. sorafenib } \\
\text { group) } \\
\text { LDLT group }\end{array}$ & $0.15(0.09-0.25)$ & $<0.001$ & $0.14(0.08-0.24)$ & $<0.001$ \\
\hline
\end{tabular}

Abbreviation: HR, hazard ratio; CI, confidence interval; AJCC, American Joint Committee on Cancer; LDLT, living donor liver transplantation; IPW, inverse probability weighting

Over many years, several groups have suggested criteria to limit liver transplantation to $\mathrm{HCC}$ patients with a good prognosis. Currently, patients with HCC beyond the Milan criteria do not have equal access to DDLT as those with HCC within the Milan criteria [25]. In LDLT, the donor frequently wishes to donate, even if the recipient has HCC beyond the Milan criteria. Although LDLT is not a curative treatment, it is palliative and prolongs OS. Therefore, systems to provide the most accurate prognostic evaluation for likely participants become increasingly important. Recently, our group developed and validated a model predicting $\mathrm{HCC}$ recurrence after LDLT (the MoRAL score) for those patients beyond the Milan criteria, including those with advanced HCC, based on reproducible predictors including serum AFP and PIVKA-II levels. Patients with a high MoRAL score might have more aggressive tumor biology, leading to a worse outcome. Therefore, we suggested that LDLT should be considered as a treatment option for patients with a low MoRAL score [8].
In the current study's subgroup analyses according to MoRAL score, we found that the gain in OS and TTP for LDLT over sorafenib is more predominant in those patients with a low MoRAL score $(81.1 \%$ vs. $5.8 \%$ for 5-year OS rate, $22.2 \%$ vs. $96.8 \%$ for 5-year tumor recurrence rate), compared with those with a high MoRAL score $(28.3 \% v s .4 .3 \%$ for 5-year OS rate, $91.6 \% v s$. $95.4 \%$ months for 5-year tumor recurrence rate). Tumor recurrence after liver transplantation has a poor prognosis because immunosuppression is a well-known risk factor for tumor growth [26]. Patients with a high MoRAL score have aggressive tumor biology, leading to a high tumorrecurrence rate. Therefore, the gain in TTP for a patient with a high MoRAL score is lower than that for a patient with a low MoRAL score, leading to a smaller gain in OS. However, it is worth noting that among those patients with a high MoRAL score, LDLT is still superior to sorafenib in OS and TTP. For a patient with a high MoRAL score, the gain in OS and TTP is lower than that seen in a patient with a low MoRAL score, but it is still better than 
sorafenib, the current standard treatment. Therefore, LDLT might be considered a treatment option for patients with a high MoRAL score.

In the multivariate analysis, AJCC T classification did not stand out as a prognostic factor for OS and TTP. The LDLT group had more advanced intrahepatic tumors (higher $\mathrm{T}$ classification) than the sorafenib group at baseline and AJCC T classification was a confounding factor for the treatment group $(P<0.001)$. Actually, there was a significant association between AJCC T classification and OS in univariate analysis. If we omit treatment group from multivariate analysis, $T$ classification was an independent prognostic factor for both OS and TTP (data not shown). It seems that $\mathrm{T}$ classification failed to become an independent prognostic factor in the multivariate analysis (both before and after IPW), probably because treatment group (LDLT vs. sorafenib) was the strongest independent prognostic factor and there was a confounding effect between treatment group and $\mathrm{T}$ classification.

Our study has several limitations. First, this study was performed in Korea, where $70 \%$ to $80 \%$ of HCC patients are infected with hepatitis B virus [27]. Therefore, our results might not be generalizable, since most Western countries have HCC patients with different underlying etiologies such as hepatitis $\mathrm{C}$ virus infection. Second, there might be an ethical issue in performing liver transplantation in beyond the Milan criteria patients. However, we followed ethical principles, including autonomy and nonmaleficence [28]. Third, this was a retrospective cohort study with inherent limitations

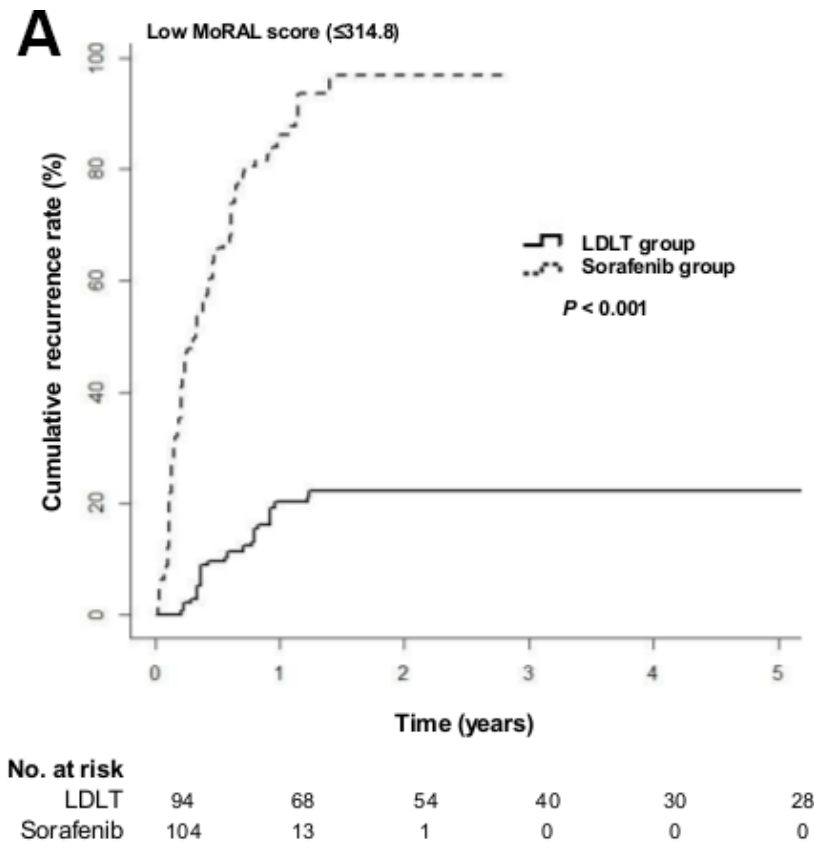

including selection bias; we therefore performed IPW to minimize bias. However, some unmeasured difference may not be balanced. Further prospective studies are necessary to confirm superior treatment outcome of LDLT over sorafenib.

In conclusion, for HCC patients beyond the Milan criteria, LDLT demonstrates significantly longer OS and TTP than sorafenib. Therefore, patients beyond the Milan criteria with a low MoRAL score and without extrahepatic metastasis might be good candidates for LDLT rather than sorafenib treatment, if there is a willing living related donor.

\section{MATERIALS AND METHODS}

\section{Study subjects}

We retrospectively analyzed consecutive patients who were treated with either LDLT or sorafenib between June 2005 and December 2014 at Seoul National University Hospital, Seoul, Korea. The diagnosis of HCC was based on the noninvasive criteria of the American Association for the Study of Liver Diseases or on the histologic diagnosis [29]. All donors received psychiatric counseling to determine whether they were suitably motivated to undergo LDLT. In both LDLT and sorafenib groups, patients were with $\mathrm{HCC}$ which was unresectable and contraindicated or refractory to transarterial chemoembolization. In patients with HCC beyond the Milan criteria, LDLT was performed only for those who were willing to undergo LDLT. None of the patients in

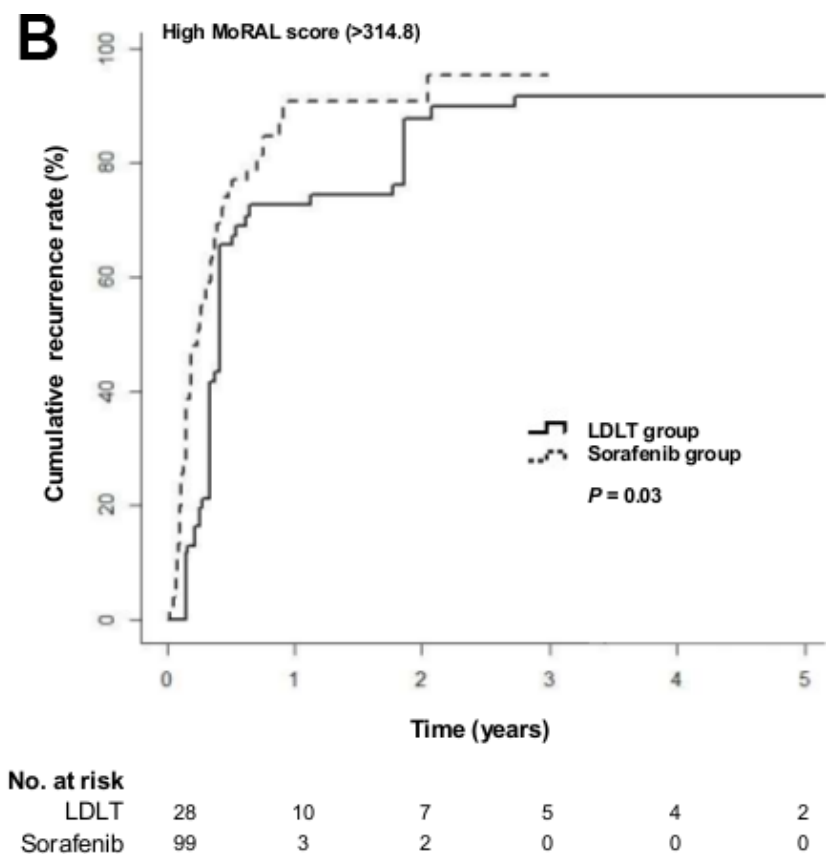

Figure 4: Cumulative tumor recurrence rates in the LDLT and sorafenib groups according to the MoRAL score using a cut-off of 314.8. (A) Cumulative tumor recurrence rates in patients with a low MoRAL score $(\leq 314.8 ; P<0.001$ by log-rank test) (B) Cumulative tumor recurrence rates in patients with a high MoRAL score $(>314.8 ; P=0.03$ by log-rank test). 
the LDLT group were treated with sorafenib prior to liver transplantation. Patients with extrahepatic metastasis and hepatic vein invasion were excluded. In the sorafenib group, patients received oral sorafenib $400 \mathrm{mg}$ twice daily. Treatment with sorafenib continued until either unacceptable toxicity or disease progression occurred.

Pretreatment staging included dynamic liver computed tomography (CT), magnetic resonance imaging, positron emission tomography-CT, bone scanning, and lowdose chest CT. The demographic findings included patient sex, age, viral status, pretransplant AFP and PIVKA-II levels, Child-Pugh class, tumor size, and number of tumors according to pretransplant imaging studies; the presence of portal vein invasion was also noted.

The safety of the living donor was of primary concern. All LDLTs were performed after obtaining fully informed written consent and multidisciplinary approval including internal medicine, surgery, psychiatry, and radiology. The study protocol conformed to the ethical guidelines of the World Medical Association Declaration of Helsinki and was approved by the institutional review board of Seoul National University Hospital (IRB No. 1305-561-490).

\section{Assessment}

Data on clinical and laboratory findings were collected retrospectively from all patients by reviewing their electronic medical records. One radiologist independently reviewed all radiologic images, blinded to survival data, to determine the number and size of tumors, presence or absence of vascular invasion, treatment response, and recurrence. In the LDLT group, patients underwent dynamic CT or magnetic resonance imaging every 2 to 4 months for the first 2 years after liver transplantation and every 3 to 6 months thereafter. In the sorafenib group, patients underwent dynamic CT or magnetic resonance imaging every 4 to 6 weeks during treatment, and adverse events were assessed during the first 3 to 4 weeks of therapy. Patients who discontinued sorafenib treatment due to severe adverse events were not included in the analysis.

The primary endpoint was OS, defined as the time from the date of diagnosis until the date of death from any cause. The secondary endpoints included TTP.

\section{Statistical analysis}

The baseline patient characteristics were expressed as the median (range). To minimize selection bias and better describe the treatment effect of different modalities, baseline characteristics were balanced using IPW. Propensity scores were calculated using a logistic regression model. We predicted the probability for each patient on the basis of the pretreatment variables. After the inverse probabilities of the propensity score weight was generated, the 2 groups were balanced using IPW.
The Kaplan-Meier method was used to estimate OS and TTP. Multivariate Cox proportional hazards regression analysis was performed to compute the HR. Patients were divided into two groups according to the MoRAL score using a cutoff of 314.8. MoRAL-score subgroup analysis was then performed to compare the LDLT and sorafenib groups.

Statistical analyses were performed using PASW STATISTICS 22.0 (SPSS Inc., Chicago, IL, USA) and $\mathrm{R}$ language, version 3.01 (R Foundation for Statistical Computing, Vienna, Austria). All statistical tests were 2-sided and conducted in an explorative manner with a significance level of 0.05 .

\section{Abbreviations}

HCC, hepatocellular carcinoma; LDLT, living donor liver transplantation; DDLT, deceased-donor liver transplantation; MoRAL, model to predict tumor recurrence after LDLT; AFP, alpha-fetoprotein; PIVKAII, protein induced by vitamin $\mathrm{K}$ absence-II; OS, overall survival; TTP, time-to-progression; CT, computed tomography; HR, hazard ratio; CI, confidence interval; IPW, inverse probability weighting; AJCC, American Joint Committee on Cancer; BCLC, Barcelona clinic liver cancer; MELD, model for end-stage liver disease; IQR, interquartile range.

\section{CONFLICTS OF INTEREST}

The authors declare no conflicts of interest.

\section{FUNDING}

This study was funded by grant from the National R\&D Program for Cancer Control, Ministry for Health and Welfare, Republic of Korea (1420050), Seoul National University Hospital Research Funds (03-20160380 and 06-2016-2880), and grant from Liver Research Foundation of Korea as part of Bio Future Strategies Research Project.

\section{REFERENCES}

1. Mazzaferro V, Regalia E, Doci R, Andreola S, Pulvirenti A, Bozzetti F, Montalto F, Ammatuna M, Morabito A, Gennari L. Liver transplantation for the treatment of small hepatocellular carcinomas in patients with cirrhosis. N Engl J Med. 1996; 334:693-699.

2. Wiesner R, Edwards E, Freeman R, Harper A, Kim R, Kamath P, Kremers W, Lake J, Howard T, Merion RM, Wolfe RA, Krom R, United Network for Organ Sharing Liver Disease Severity Score C. Model for end-stage liver disease (MELD) and allocation of donor livers. Gastroenterology. 2003; 124:91-96. 
3. Ringe B, Pichlmayr R, Wittekind C, Tusch G. Surgical treatment of hepatocellular carcinoma: experience with liver resection and transplantation in 198 patients. World J Surg. 1991; 15:270-285.

4. Marsh JW, Dvorchik I. Liver organ allocation for hepatocellular carcinoma: are we sure? Liver Transpl. 2003; 9:693-696.

5. Choi HJ, Kim DG, Na GH, Hong TH, You YK. Extended criteria for living donor liver transplantation in patients with advanced hepatocellular carcinoma. Transplant Proc. 2012; 44:399-402.

6. Gondolesi GE, Roayaie S, Munoz L, Kim-Schluger L, Schiano T, Fishbein TM, Emre S, Miller CM, Schwartz ME. Adult living donor liver transplantation for patients with hepatocellular carcinoma: extending UNOS priority criteria. Ann Surg. 2004; 239:142-149.

7. Chen CL, Cheng YF, Yu CY, Ou HY, Tsang LL, Huang TL, Chen TY, Concejero A, Wang CC, Wang SH, Lin TS, Liu YW, Yang CH, et al. Living donor liver transplantation: the Asian perspective. Transplantation. 2014; 97:S3.

8. Lee JH, Cho Y, Kim HY, Cho EJ, Lee DH, Yu SJ, Lee JW, Yi NJ, Lee KW, Kim SH, Kim JM, Joh JW, Teperman LW, et al. Serum Tumor Markers Provide Refined Prognostication in Selecting Liver Transplantation Candidate for Hepatocellular Carcinoma Patients Beyond the Milan Criteria. Ann Surg. 2016; 263:842-850.

9. Llovet JM, Ricci S, Mazzaferro V, Hilgard P, Gane E, Blanc JF, de Oliveira AC, Santoro A, Raoul JL, Forner A, Schwartz M, Porta C, Zeuzem S, et al. Sorafenib in advanced hepatocellular carcinoma. N Engl J Med. 2008; 359:378-390.

10. Cheng AL, Guan Z, Chen Z, Tsao CJ, Qin S, Kim JS, Yang TS, Tak WY, Pan H, Yu S, Xu J, Fang F, Zou J, et al. Efficacy and safety of sorafenib in patients with advanced hepatocellular carcinoma according to baseline status: subset analyses of the phase III Sorafenib Asia-Pacific trial. Eur J Cancer. 2012; 48:1452-1465.

11. Llovet JM, Burroughs A, Bruix J. Hepatocellular carcinoma. Lancet. 2003; 362:1907-1917.

12. Bruix J, Sherman M, Practice Guidelines Committee. American Association for the Study of Liver Diseases. Management of hepatocellular carcinoma. Hepatology. 2005; 42:1208-1236.

13. Llovet JM, Bruix J. Systematic review of randomized trials for unresectable hepatocellular carcinoma: Chemoembolization improves survival. Hepatology. 2003; 37:429-442.

14. Lopez PM, Villanueva A, Llovet JM. Systematic review: evidence-based management of hepatocellular carcinoma-an updated analysis of randomized controlled trials. Aliment Pharmacol Ther. 2006; 23:1535-1547.

15. Benson AB 3rd, Abrams TA, Ben-Josef E, Bloomston PM, Botha JF, Clary BM, Covey A, Curley SA, D'Angelica MI, Davila R, Ensminger WD, Gibbs JF, Laheru D, et al. NCCN clinical practice guidelines in oncology: hepatobiliary cancers. J Natl Compr Canc Netw. 2009; 7:350-391.

16. Yu SJ. A concise review of updated guidelines regarding the management of hepatocellular carcinoma around the world: 2010-2016. Clin Mol Hepatol. 2016; 22:7-17.
17. Meng MB, Cui YL, Lu Y, She B, Chen Y, Guan YS, Zhang RM. Transcatheter arterial chemoembolization in combination with radiotherapy for unresectable hepatocellular carcinoma: a systematic review and metaanalysis. Radiother Oncol. 2009; 92:184-194.

18. Lee JM, Jang BK, Lee YJ, Choi WY, Choi SM, Chung WJ, Hwang JS, Kang KJ, Kim YH, Chauhan AK, Park SY, Tak WY, Kweon YO, et al. Survival outcomes of hepatic resection compared with transarterial chemoembolization or sorafenib for hepatocellular carcinoma with portal vein tumor thrombosis. Clin Mol Hepatol. 2016; 22:160-167.

19. Wilhelm SM, Carter C, Tang L, Wilkie D, McNabola A, Rong H, Chen C, Zhang X, Vincent P, McHugh M, Cao Y, Shujath J, Gawlak S, et al. BAY 43-9006 exhibits broad spectrum oral antitumor activity and targets the RAF/MEK/ ERK pathway and receptor tyrosine kinases involved in tumor progression and angiogenesis. Cancer Res. 2004; 64:7099-7109.

20. Cheng AL, Kang YK, Chen Z, Tsao CJ, Qin S, Kim JS, Luo R, Feng J, Ye S, Yang TS, Xu J, Sun Y, Liang H, et al. Efficacy and safety of sorafenib in patients in the AsiaPacific region with advanced hepatocellular carcinoma: a phase III randomised, double-blind, placebo-controlled trial. Lancet Oncol. 2009; 10:25-34.

21. Pons F, Varela M, Llovet JM. Staging systems in hepatocellular carcinoma. HPB (Oxford). 2005; 7:35-41.

22. Chan AT, Kishi Y, Chan SL, Vauthey JN. Accomplishments in 2007 in the management of hepatobiliary cancers. Gastrointest Cancer Res. 2008; 2:S25-31.

23. Yau T, Tang VY, Yao TJ, Fan ST, Lo CM, Poon RT. Development of Hong Kong Liver Cancer staging system with treatment stratification for patients with hepatocellular carcinoma. Gastroenterology. 2014; 146:1691-1700 e1693.

24. Befeler AS, Hayashi PH, Di Bisceglie AM. Liver transplantation for hepatocellular carcinoma. Gastroenterology. 2005; 128:1752-1764.

25. Yao FY. Liver transplantation for hepatocellular carcinoma: beyond the Milan criteria. Am J Transplant. 2008; 8:1982-1989.

26. Vivarelli M, Cucchetti A, Piscaglia F, La Barba G, Bolondi L, Cavallari A, Pinna AD. Analysis of risk factors for tumor recurrence after liver transplantation for hepatocellular carcinoma: key role of immunosuppression. Liver Transpl. 2005; 11:497-503.

27. Huh K, Choi SY, Whang YS, Lee DS. Prevalence of viral hepatitis markers in Korean patients with hepatocellular carcinoma. J Korean Med Sci. 1998; 13:306-310.

28. Miller CM, Smith ML, Diago Uso T. Living donor liver transplantation: ethical considerations. Mt Sinai J Med. 2012; 79:214-222.

29. Bruix J, Sherman M, American Association for the Study of Liver D. Management of hepatocellular carcinoma: an update. Hepatology. 2011; 53:1020-1022. 